\title{
Prospective study on the safety and feasibility of early ileostomy closure 2 weeks after lower anterior resection for rectal cancer
}

\author{
Kyung Ha Lee ${ }^{1}$, Hyung Ook Kim², Jin Soo Kim¹, Ji Yeon Kim \\ ${ }^{1}$ Department of Surgery, Chungnam National University Hospital, Daejeon, Korea \\ 2Department of Surgery, Kangbuk Samsung Hospital, Seoul, Korea
}

\begin{abstract}
Purpose: Transient loop ileostomies in rectal cancer surgery are generally closed after 2 or more months to allow adequate time for anastomotic healing. Maintaining the ileostomy may cause medical, surgical, or psychological complications; it also reduces the quality of life, and increase treatment costs. We performed this study to evaluate the safety and feasibility of early ileostomy closure 2 weeks postoperatively.

Methods: If a patient who underwent total mesorectal excision had 2 or more risk factors for anastomotic leakage, a loop ileostomy was created. After confirmation of intact anastomosis via sigmoidoscopy and proctography 1 week postoperatively, the patient was enrolled and ileostomy was closed 2 weeks postoperatively. The primary endpoint was the frequency of complication after ileostomy repair.

Results: Thirty patients were enrolled in the study and 6 were excluded due to anastomotic leakage. Except for 1 case of wound infection (4.2\%), no patient experienced any complication including newly developed leakage after the ileostomy closure. The mean duration to repair was 13.1 days (range, 8-16 days) and mean duration to the start of adjuvant treatment after radical surgery was 5.37 weeks (range, 3.0-8.1 weeks).

Conclusion: Transient loop ileostomy, which is confirmed to be intact endoscopically and radiologically, can be safely closed 2 weeks postoperatively without requiring a significant delay in adjuvant chemotherapy.

[Ann Surg Treat Res 2019;96(1):41-46]
\end{abstract}

Key Words: Ileostomy, Feasibility studies

\section{INTRODUCTION}

Transient ileostomy is selectively performed for rectal cancer surgery with risk of anastomotic failure. Although ileostomy cannot prevent leakage itself [1,2], a temporary ileostomy can minimize the aggravation of clinical symptoms due to leakage and convert major complications that demand surgical intervention to minor complications that can improve with conservative treatment [3,4]. Transient ileostomies are generally closed 2 or more months after radical surgery and stoma creation, this allows adequate time for the anastomosis to fully heal [5]. However, some patients sometimes need to maintain ileostomies for more than 6 months, until adjuvant treatment is terminated completely. Ileostomy maintenance can cause medical complications including dehydration, electrolyte imbalance, malnutrition, or dermatologic problem at the parastomal area, as well as surgical complications including ileus, obstruction, or herniation. It also disturbs the activities of daily living, affects the quality of life, and sometimes produces psychological complications, such as lowered self-esteem and
Received April 3, 2018, Revised July 25, 2018, Accepted July 26, 2018

Corresponding Author: Ji Yeon Kim

Department of Surgery, Chungnam National University Hospital, 282

Munhwa-ro, Jung-gu, Daejeon 35015, Korea

Tel: +82-42-280-7175, Fax: +82-42-257-8024

E-mail: jkim@cnu.ac.kr

ORCID code: https://orcid.org/0000-0001-7964-5060
Copyright (c) 2019, the Korean Surgical Society

(c) Annals of Surgical Treatment and Research is an Open Access Journal. All articles are distributed under the terms of the Creative Commons Attribution NonCommercial License (http://creativecommons.org/licenses/by-nc/4.0/) which permits unrestricted non-commercial use, distribution, and reproduction in any medium, provided the original work is properly cited. 
depression. Patients are also required to visit the hospital periodically and pay for supplies [6,7]. These complications could be reduced by earlier closure of transient ileostomy in patients who do not need to maintain the ileostomy because no anastomotic complications have occurred after stoma creation. Although there are some studies regarding early ileostomy closure, there was no study about the safety and feasibility of early ileostomy closure in domestic circumstances, and to the best of our knowledge, no study examined its effect on adjuvant treatment [8-10]. Therefore, the present study aimed to evaluate the safety and feasibility of early ileostomy closure 2 weeks after total mesorectal excision (TME) and stoma creation in patients with risk factors for leakage.

\section{METHODS}

\section{Study population}

Patients between 20 and 90 years of age who underwent TME with transient ileostomy for rectal cancer and did not have any anastomotic complications for 1 week postoperatively were enrolled. The creation of a transient ileostomy was indicated if the patient had 2 or more of the following risk factors for anastomotic leakage: male sex, lower rectal cancer (distal border of tumor below $5 \mathrm{~cm}$ from the anal verge), preoperative radiotherapy, 3 or more staples for rectal division, and positive findings on an intraoperative leakage test with air. These criteria were strictly applied to avoid the creating an unnecessary ileostomy for the study itself. Enrolled patients underwent sigmoidoscopy and proctography approximately 1 week after surgery. To allow precise inspection without disturbance to the remnant gastrograffin in the rectum, a flexible sigmoidoscopy was performed first, followed by proctography. The observation range of proctography was confined to the anastomoses and neorectum and water soluble gastrograffin was used instead of barium, because barium can aggravate an inflammatory reaction if it leaks into the peritoneal cavity. If the anastomosis was confirmed intact in both of examinations, the ileostomy was closed 2 weeks postoperatively. If a visible defect on sigmoidoscopy or leakage of contrast media on proctography was found, the patient was excluded from analysis. Other exclusion criteria were: complication of Clavien-Dindo classification (CDC) III or more [11] that was unrelated to the ileostomy; inappropriate general condition for an immediate second surgery and general anesthesia; refusal for early ileostomy closure. The flowchart was presented in Fig. 1, and the imaging findings of intact anastomosis and incidental leakage were presented in Figs. 2 and 3, respectively. The study was approved by the Institutional Review Board of the institute (approval number: 2015-02-016) and informed consent was obtained from all patients.

\section{Endpoints}

The primary endpoint was the frequency of complications following ileostomy repair including newly developed anastomotic leakage. The secondary endpoints were hospital stay and the time to the start of adjuvant treatment after radical surgery.

\section{Calculated sample size}

Although the frequency of complications after ileostomy closure varies across institutes, it is generally reported as approximately $30 \%$ (based on a synthesis of the results from numerous studies) [10]. Given a 10\% difference in the primary endpoint, a power of $80 \%$, and a significance level of $5 \%$, the required sample number was estimated to be 30 patients. The sample size calculation was performed using PASS 13 (Power

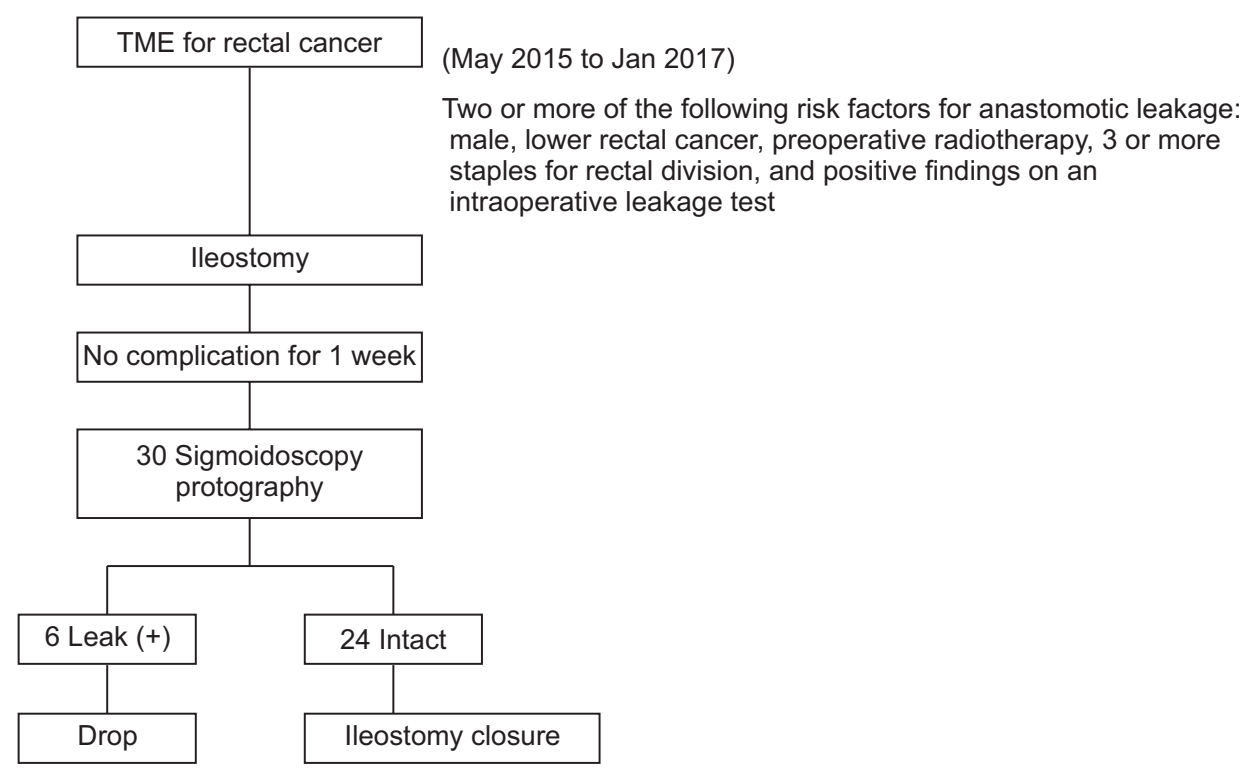

Fig. 1. The flow chart. TME, total mesorectal excision. 

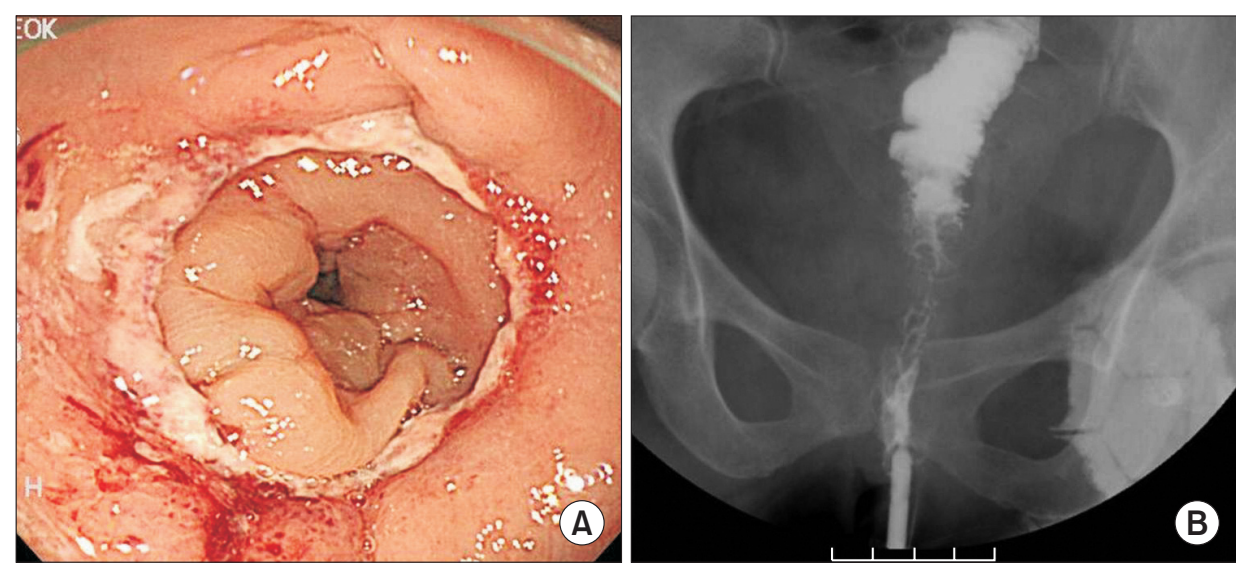

Fig. 2. Findings of intact anastomosis: flexible sigmoscopy $(\mathrm{A})$ and proctography $(\mathrm{B})$.
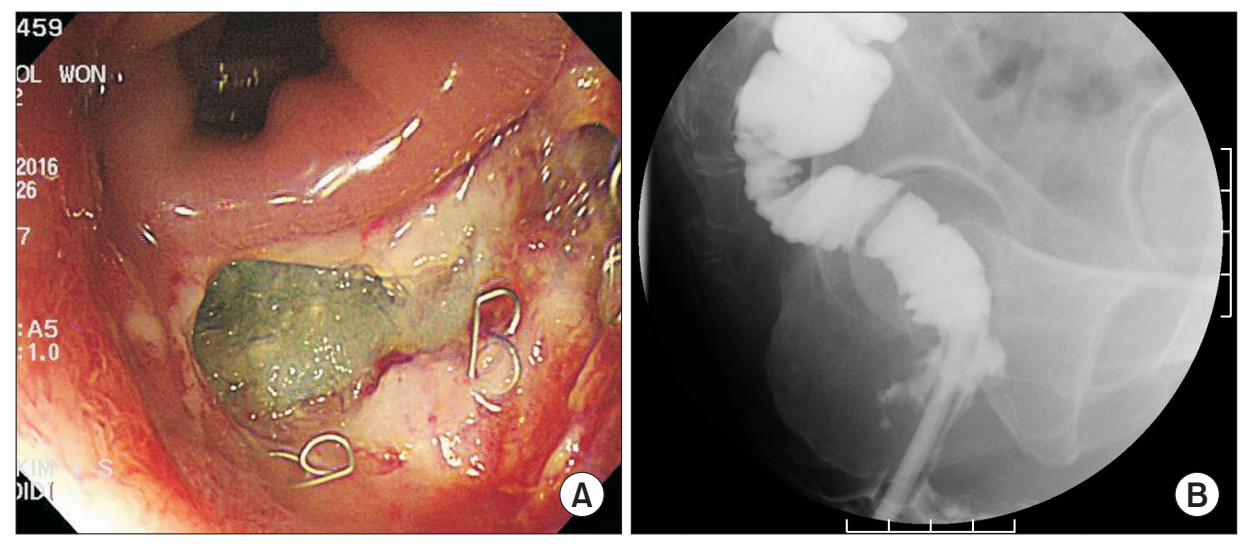

Fig. 3. Findings of incidental leakage: flexible sigmoscopy (A) and proctography (B).

Analysis and Sample Size 13, NCSS, LLC, Kaysville, UT, USA).

\section{Statistical analysis}

Descriptive statistics were performed using IBM SPSS Statistics ver. 20.0 (IBM Co., Armonk, NY, USA).

\section{RESULTS}

Thirty patients who underwent TME and transient loop ileostomy between May 2015 and January 2017 were initially enrolled. Six patients were excluded because anastomotic leakage was found on sigmoidoscopy or proctography. All of those patients did not show clinically significant manifestation owing to ileostomy, and reevaluation and ileostomy closure were performed according to their each personal status. Finally, 24 patients were analyzed. Characteristics of the patients and the radical surgery are presented in Tables 1 and 2, respectively. Characteristics of the pathology and ileostomy closure surgery are presented in Tables 3 and 4, respectively. Except for one case of wound infection of CDC II managed with CuraVAC (Daewoong Pharm. Co., Ltd., Seoul, Korea) (4.2\%), no patient experienced any complication including newly developed leakage after the ileostomy closure. The mean duration between radical surgery and evaluation was 10.5 days (range, 6-14 days), and the mean
Table 1. Patient characteristics

\begin{tabular}{lc}
\hline \multicolumn{1}{c}{ Characteristic } & Value \\
\hline Sex & \\
Male & $18(75.0)$ \\
Female & $6(25.0)$ \\
Age (yr) & $64.7(35-83)$ \\
Tumor location & \\
Lower rectum (below $5 \mathrm{~cm}$ from the anal verge) & $11(41.7)$ \\
Middle rectum (5-10 cm from anal verge) & $13(54.2)$ \\
Preoperative treatment & $10(41.7)$ \\
None & $12(50.0)$ \\
Neoadjuvant concurrent chemoradiotherapy & $1(4.2)$ \\
Neoadjuvant short course radiotherapy with & \\
chemotherapy & $1(4.2)$ \\
Palliative chemotherapy
\end{tabular}

Values are presented as number (\%) or mean (range).

duration between radical surgery and ileostomy closure was 13.1 days (range, 8-16 days). The mean hospital stay was 20.2 days (range, 11-34 days), and the mean duration between radical surgery and the start of adjuvant treatment was 5.37 weeks (range, 3.0-8.1 weeks). 
Table 2. Characteristics of radical surgery

\begin{tabular}{lr}
\multicolumn{1}{c}{ Variable } & Number $(\%)$ \\
\hline $\begin{array}{l}\text { Operation name of radical surgery } \\
\text { Low anterior resection }\end{array}$ & $21(87.5)$ \\
Intersphincteric resection with colo-anal & $3(12.5)$ \\
$\quad$ anastomosis & \\
No. of staples ${ }^{\text {a) }}$ & \\
$\quad$ None & $3(12.5)$ \\
1 & $1(4.2)$ \\
2 & $17(70.8)$ \\
3 & $3(12.5)$ \\
Intraoperative leakage test & $14(58.3)$ \\
$\quad$ Negative & $10(41.7)$ \\
$\quad$ Not done (for cases with enough risk factors & \\
$\quad$ preoperatively) & \\
Postoperative complication & $23(95.8)$ \\
$\quad$ None & $1(4.2)$ \\
$\quad$ Pelvic abscess (CDC IIla, without anastomotic \\
leakage)
\end{tabular}

CDC, Clavien-Dindo classification.

${ }^{a}$ For cases without stapling, anastomosis was performed manually with $3 / 0$ absorbable suture. For cases with one stapling, $60-\mathrm{mm}$ cartilage was used. For cases with 2 or 3 stapling, $45-\mathrm{mm}$ cartiliages were used.

Table 3. Pathologic characteristics

\begin{tabular}{cc}
\hline Variable & Number $(\%)$ \\
$\mathrm{pT}$ & $2(12.5)$ \\
0 & $4(12.5)$ \\
1 & $8(33.3)$ \\
2 & $9(37.5)$ \\
3 & $1(4.2)$ \\
4 & \\
$\mathrm{pN}$ & $18(75.0)$ \\
0 & $4(16.7)$ \\
1 & $2(8.3)$ \\
2 & \\
$\mathrm{pM}$ & $21(87.5)$ \\
0 & $3(12.5)$ \\
\hline
\end{tabular}

\section{DISCUSSION}

Collagen synthesis for colonic anastomotic healing begins to increase 3 hours after surgery, attains a 6-fold maximal stimulation 4 days after surgery, and returns to preoperative levels 4 weeks after surgery [12]. Colonic anastomosis has a greater tendency towards failure compared to surgery for the small bowel, a lower degree of collagen synthesis in the colonic wound is thought to be a significant contributing factor [12]. Therefore, anastomotic failure remains the most important and fatal complication of colorectal cancer surgery. Although
Table 4. Characteristics of ileostomy repair surgery

\begin{tabular}{lc}
\hline \multicolumn{1}{c}{ Variable } & Value \\
\hline $\begin{array}{l}\text { Duration between radical surgery and } \\
\text { evaluation (day) }\end{array}$ & $10.5(6-14)$ \\
$\begin{array}{l}\text { Duration between radical surgery and } \\
\text { ileostomy repair (day) }\end{array}$ & $13.1(8-16)$ \\
Operation time (min) & $82.95(50-110)$ \\
Anastomosis & $21(87.5)$ \\
$\quad$ Manual & $3(12.5)$ \\
Stapler & $23(70.8)$ \\
Postoperative complication & $1(4.2)$ \\
$\quad$ None & \\
Wound infection (CDC II) &
\end{tabular}

Values are presented as mean (range) or number (\%).

CDC, Clavien-Dindo classification.

a temporary ileostomy is created to prevent fatal situations, including peritonitis or consequent sepsis, many patients who undergo ileostomy have intact anastomoses. In such patients, the ileostomy does not necessary need to be maintained after the confirmation of an intact anastomosis. The precise time when a surgeon can conclude that there is no anastomotic failure varies as the healing process is not identical across patients. There are numerous risk factors for anastomotic leakage (e.g., sex, tumor location, irradiation, or multiple stapling for the intraoperative rectal division). Considering these differences, the exact timing of ileostomy closure in patients with intact anastomosis is challenging to determine. As mentioned above, the time required for anastomotic healing is at least four days if no additional problems occur. However, predicting the timing of anastomotic healing is challenging when the patient has risk factors. It is commonly believed that more than 2 months are required for the stabilization of the anastomosis, therefore, most ileostomies are conventionally closed minimally 2 months postoperatively. However, some surgeons have recently asserted that early ileostomy closure within 2 weeks is safe.

Perez et al. [5] reported that complications were significantly associated with shorter intervals between the primary surgery and ileostomy closure, and concluded that ileostomy closure must be performed more than 8.5 weeks postoperatively. However, as the concept of minimally invasive treatment has become accepted, and interest in the quality of life has increased, research demonstrating the safety of early ileostomy closure has been reported. For example, a nonrandomized prospective study by Menegaux et al. [8] demonstrated that the ileostomy could be closed 10 days postoperatively in selected healthy patients. Similarly, a nonrandomized prospective study by Bakx et al. [9] showed that early ileostomy closure 1 to 3 weeks postoperatively was feasible, with low morbidity and no mortality. Furthermore, a randomized clinical trial by Alves et al. [13] demonstrated that early ileostomy closure 
8 days postoperatively is feasible in selected patients, with reduced hospital stays and postoperative complications. Recently, Danielsen et al. [14] reported the results of a multicenter randomized controlled trial with 127 patients, demonstrating the safety of early ileostomy closure 8 to 13 days postoperatively.

In the present study, transient ileostomy was performed only in patients with 2 or more risk factors for leakage, and the mean duration between radical surgery and ileostomy closure was 13.1 days (range, 8-14 days). All patients agreed to participate in the study, and there were no complications resulting from the decreased interval between radical surgery and ileostomy closure.

Reoperation in the immediate postoperative period has been conventionally avoided, since dense and inflammatory adhesions are maximum in this period [15]. To evaluate the surgical feasibility related to postoperative adhesion, we evaluated the operation time. The difference was not significant compared to the time generally required for conventional ileostomy closure.

Wound infection was the only complication in the present study, with a rate of $4.2 \%$ ( 1 case). Wound infections are a relatively common complication due to bacterial colonization in the skin around the ileostomy, with a reported rate of $1.5 \%-5 \%$ $[13,16,17]$. Although recovery or immunity is possibly reduced in the immediate postoperative period, and immune defense mechanism alterations after major surgery or trauma are known to render the host susceptible to infectious complications via diverse cytokine activities [18], the wound infection rate after early ileostomy closure is considered to be almost equal to that after conventional ileostomy closure.

Many patients suffer low anterior resection syndrome, which severly impairs the quality of life, after rectal cancer surgery. Although the avoidance of this complication during the immediate postoperative period can be an additional benefit of ileostomy, most patients will eventually experience it after ileostomy closure. Therefore, it is rare to maintain ileostomy only to prevent low anterior resection syndrome when the anastomosis is intact, and satisfaction with ileostomy closure can outweigh the discomfort due to low anterior resection syndrome. However, if the low anterior resection syndrome is severe, the patient may require more time to recover before the start of adjuvant treatment, delaying adjuvant treatment.

In the present study, the mean duration between radical surgery and the start of chemotherapy was 5.37 weeks (range,
3.0-8.1 weeks). Adjuvant treatment was performed in 14 out of 24 patients; 10 patients did not undergo adjuvant treatment (7 patients with early rectal cancer, 2 patients with ypCR, and 1 older patient who refused adjuvant treatment). In 10 patients, adjuvant treatment was started within 6 weeks of radical surgery. Given that a delay in adjuvant chemotherapy after colorectal cancer resection beyond 6-8 weeks is associated with inferior oncologic outcomes [19,20], early ileostomy closure is considered to not significantly prolong the duration between radical surgery and the start of chemotherapy. Thus there is little chance that early ileostomy closure deteriorates the oncological prognosis. However, in 4 patients, adjuvant chemotherapy was delayed (21.4\%). One patient underwent 14 cycles of palliative chemotherapy before radical surgery; thus, he wanted more recovery time and refused to restart chemotherapy in a timely manner. His reason for the refusal of chemotherapy was related to his general condition, impaired by the previous long period of chemotherapy, and was not related to the early ileostomy closure. One patient required treatment for an ileostomy closure wound. Wound problems are considered to be equal between conventional and early ileostomy closure. Therefore, the relationship between the delay of chemotherapy and the timing of ileostomy closure is unclear in this case. Two patients wanted more recovery time because of the presence of low anterior resection syndrome. In such cases, chemotherapy might not be delayed if it started before ileostomy closure. Considering these 2 patients, the possibility that early ileostomy closure may delay adjuvant chemotherapy should be considered, and care should be taken to prevent excessive delay in adjuvant treatment, especially for patients with adjuvant treatment priority due to locally or metastatically advanced disease.

The present study was noncomparative and the number of patients was relatively small. Further comparative research with large sample sizes is necessary to evaluate oncological results following early ileostomy closure.

In conclusion, transient loop ileostomy, which is confirmed to be intact endoscopically and radiologically, can be safely closed 2 weeks postoperatively without requiring a significant delay in adjuvant chemotherapy.

\section{CONFLICTS OF INTEREST}

No potential conflict of interest relevant to this article was reported.

\section{REFERENCES}

1. Mealy K, Burke P, Hyland J. Anterior

resection without a defunctioning colos- tomy: questions of safety. Br J Surg 1992; 
79:305-7.

2. Machado M, Hallbook O, Goldman S, Nystrom PO, Jarhult J, Sjodahl R. Defunctioning stoma in low anterior resection with colonic pouch for rectal cancer: a comparison between two hospitals with a different policy. Dis Colon Rectum 2002;45:940-5.

3. Matthiessen P, Hallbook O, Rutegard J, Simert G, Sjodahl R. Defunctioning stoma reduces symptomatic anastomotic leakage after low anterior resection of the rectum for cancer: a randomized multicenter trial. Ann Surg 2007;246:207-14.

4. Huser N, Michalski CW, Erkan M, Schuster T, Rosenberg R, Kleeff J, et al. Systematic review and meta-analysis of the role of defunctioning stoma in low rectal cancer surgery. Ann Surg 2008;248: 52-60.

5. Perez RO, Habr-Gama A, Seid VE, Proscurshim I, Sousa AH Jr, Kiss DR, et al. Loop ileostomy morbidity: timing of closure matters. Dis Colon Rectum 2006;49:153945.

6. O'Leary DP, Fide CJ, Foy C, Lucarotti ME. Quality of life after low anterior resection with total mesorectal excision and temporary loop ileostomy for rectal carcinoma. Br J Surg 2001;88:1216-20.

7. Tsunoda A, Tsunoda Y, Narita K, Watanabe M, Nakao K, Kusano M. Quality of life after low anterior resection and temporary loop ileostomy. Dis Colon
Rectum 2008;51:218-22.

8. Menegaux F, Jordi-Galais P, Turrin N, Chigot JP. Closure of small bowel stomas on postoperative day 10. Eur J Surg 2002; 168:713-5.

9. Bakx R, Busch OR, van Geldere D, Bemelman WA, Slors JF, van Lanschot JJ. Feasibility of early closure of loop ileostomies: a pilot study. Dis Colon Rectum 2003:46:1680-4.

10. Hindenburg T, Rosenberg J. Closing a temporary ileostomy within two weeks. Dan Med Bull 2010;57:A4157.

11. Clavien PA, Barkun J, de Oliveira ML, Vauthey JN, Dindo D, Schulick RD, et al. The Clavien-Dindo classification of surgical complications: five-year experience. Ann Surg 2009;250:187-96.

12. Martens MF, Hendriks T. Postoperative changes in collagen synthesis in intestinal anastomoses of the rat: differences between small and large bowel. Gut 1991; 32:1482-7.

13. Alves A, Panis Y, Lelong B, Dousset B, Benoist S, Vicaut E. Randomized clinical trial of early versus delayed temporary stoma closure after proctectomy. Br J Surg 2008:95:693-8.

14. Danielsen AK, Park J, Jansen JE, Bock D, Skullman S, Wedin A, et al. Early closure of a temporary ileostomy in patients with rectal cancer: a multicenter randomized controlled trial. Ann Surg 2017;265:28490.
15. Goussous N, Kemp KM, Bannon MP, Kendrick ML, Srvantstyan B, Khasawneh MA, et al. Early postoperative small bowel obstruction: open vs laparoscopic. Am J Surg 2015;209:385-90.

16. Wong KS, Remzi FH, Gorgun E, Arrigain S, Church JM, Preen M, et al. Loop ileostomy closure after restorative proctocolectomy: outcome in 1,504 patients. Dis Colon Rectum 2005;48:243-50.

17. Chow A, Tilney HS, Paraskeva P, Jeyarajah S, Zacharakis E, Purkayastha S. The morbidity surrounding reversal of defunctioning ileostomies: a systematic review of 48 studies including 6,107 cases. Int J Colorectal Dis 2009;24:711-23.

18. Hensler T, Hecker H, Heeg K, Heidecke CD, Bartels H, Barthlen W, et al. Distinct mechanisms of immunosuppression as a consequence of major surgery. Infect Immun 1997;65:2283-91.

19. Sun Z, Adam MA, Kim J, Nussbaum DP, Benrashid E, Mantyh CR, et al. Determining the optimal timing for initiation of adjuvant chemotherapy after resection for stage II and III colon cancer. Dis Colon Rectum 2016;59:87-93.

20. Biagi JJ, Raphael MJ, Mackillop WJ, Kong W, King WD, Booth CM. Association between time to initiation of adjuvant chemotherapy and survival in colorectal cancer: a systematic review and metaanalysis. JAMA 2011;305:2335-42. 